\title{
Principal parametric resonance of axially accelerating viscoelastic beams: Multi-scale analysis and differential quadrature verification
}

\author{
Li-Qun Chen ${ }^{\mathrm{a}, \mathrm{b}, *}$, Hu Ding ${ }^{\mathrm{a}}$ and C.W. Lim ${ }^{\mathrm{c}}$ \\ ${ }^{a}$ Department of Mechanics, Shanghai Key Laboratory of Mechanics in Energy Engineering, Shanghai University, \\ Shanghai, China \\ ${ }^{\mathrm{b}}$ Shanghai Institute of Applied Mathematics and Mechanics, Shanghai, China \\ ${ }^{\mathrm{c}}$ Department of Building and Construction, City University of Hong Kong, Kowloon, Hong Kong, China
}

Received 25 May 2010

Revised 28 July 2011

\begin{abstract}
Transverse non-linear vibration is investigated in principal parametric resonance of an axially accelerating viscoelastic beam. The axial speed is characterized as a simple harmonic variation about a constant mean speed. The material time derivative is used in the viscoelastic constitutive relation. The transverse motion can be governed by a non-linear partial-differential equation or a non-linear integro-partial-differential equation. The method of multiple scales is applied to the governing equations to determine steady-state responses. It is confirmed that the mode uninvolved in the resonance has no effect on the steady-state response. The differential quadrature schemes are developed to verify results via the method of multiple scales. It is demonstrated that the straight equilibrium configuration becomes unstable and a stable steady-state emerges when the axial speed variation frequency is close to twice any linear natural frequency. The results derived for two governing equations are qualitatively the same, but quantitatively different. Numerical simulations are presented to examine the effects of the mean speed and the variation of the amplitude of the axial speed, the dynamic viscosity, the non-linear coefficients, and the boundary constraint stiffness on the instability interval and the steady-state response amplitude.
\end{abstract}

Keywords: Vibration, nonlinearity, axially accelerating beam, viscoelasticity, method of multiple scales, differential quadrature method

\section{Introduction}

Many engineering devices can be modeled as an axially moving beam, which is a typical gyroscopic continuum. Under certain excitations, axially moving beams undergo transverse motion. If the motion amplitude is large, the non-linearity should be taken into account. Much research has been done on non-linear vibration of axially moving beams [1-26].

As exact solutions are usually unavailable for non-linear differential equations, approximate analytical methods are widely applied to investigate non-linear vibration of axially moving beams. The used methods includes the asymptotic method of Krylov, Bogoliubov, and Mitropolsky [1], the Lindstedt-Poincaré method [2,17], the method of normal forms [2,9], the method of non-linear, complex modes [4,5], the method of multiple scales [7,8,14,20-23],

\footnotetext{
*Corresponding author. E-mail: lqchen @ staff.shu.edu.cn.
} 
the incremental harmonic balance method [16], and asymptotic perturbation analysis [24]. In the analysis, the governing equations of axially moving beams are usually truncated into a discrete system containing a few modes involved the resonance. The effects of other modes have not been mathematically treated, although they were physically assumed to be neglectable because the resonance modes dominate the response.

In spite of the fact that there have been many approximate analytical investigations on non-linear vibration of axially moving beams, there are very limited researches on the topic via the direct numerical approaches. The Galerkin method is widely used in the numerical investigations $[6,10-13,19]$. Consequently, the results obtained via approximate analytical approaches were rarely verified numerically. The known exceptions are that Pellicano and Zirilli [2] and Chen and Ding [22] confirmed their analytical results via a finite difference scheme.

To address the lack of research in the above-mentioned two aspects, the present investigation studies the principal parametric resonance of an axially accelerating viscoelastic beam with the emphasis on understanding the effects of the mode uninvolved in the resonance and verifying the analytical results by the differential quadrature method. The differential quadrature method is an effective numerical technique for initial and boundary problems [24,25,27,28]. The present investigation also differentiates from the previous analytical study on axially accelerating viscoelastic beams [14] in the constitutive relation and the boundary conditions. The partial time derivative is used in the Kelvin model to describe the viscoelastic behavior of axially moving materials [29], and also used in [14]. Mockensturm and Guo [30] convincingly argued that the Kelvin model generalized to axially moving materials should contain the material time derivative to account for the energy dissipation in steady motion. Here the material time derivative is used in the Kelvin model. The beam treated analytically here is constrained by a rotating sleeve with a rotational spring at each end. The boundary conditions are more general than the simple supports used by Chen and Yang [14]. The generalization is necessary to understand some practical engineering problems. For example, if the axially moving beam models a belt on a pair of rotating pulleys, the boundary conditions at the contact points are usually not exactly a typical fixed or simply supported.

There are two kinds of non-linear transverse models of axially moving beams. One is a non-linear integro-partialdifferential equation used in $[1-6,8,9,12,14,15,18,22,25,26]$. The other is a non-linear partial-differential equation used in $[7,10,11,13,14,16-23,25,26]$. Because there has no evidence to favor any models, both models are treated in the investigation.

The present paper is organized as follows. Section 2 establishes the governing equations, a non-linear partialdifferential equation and a non-linear integro-partial-differential equation. Section 3 applies the method of multiple scales to the equations in Section 2. Section 4 presents numerical examples to demonstrate the effects of some parameters based on the analytical results. Section 5 develops a differential quadrature scheme to solve the equations in Section 2 and to predict the effects of some parameters. Section 6 compares the analytical results with numerical ones. Section 7 ends the paper with the concluding remarks.

\section{Governing equations}

A uniform axially moving viscoelastic beam, with density $\rho$, cross-sectional area $A$, moment of inertial $I$ and initial tension $P_{0}$, travels at time-dependent axial transport speed $\gamma(t)$ between two ends separated by distance $l$. Consider only the bending vibration described by the transverse displacement $v(x, t)$, where $t$ is the time and $x$ is the axial coordinate. The Newton second law of motion yields [26]

$$
\rho A\left(v,_{t t}+\dot{\gamma} v,_{x}+2 \gamma v,_{x t}+\gamma^{2} v,_{x x}\right)=\frac{\partial}{\partial x}\left[\left(P_{0}+P\right) v,_{x}\right]-\frac{\partial^{2} M}{\partial x^{2}},
$$

where $P(x, t)$ is the axial disturbed tension and $M(x, t)$ is the bending moment. The viscoelastic material of the beam obeys the Kelvin model, with the constitution relation using the material derivative [26]

$$
P / A=E_{0} \varepsilon_{L}+\eta\left(\varepsilon_{L}, t+\gamma \varepsilon_{L}, x\right),
$$

where $E_{0}$ is the stiffness, $\eta$ is the dynamic viscosity, and $\varepsilon_{L}$ is the Lagrange strain

$$
\varepsilon_{L}=\frac{1}{2} v{ }_{x}^{2}
$$


to account for geometric non-linearity due to small but finite stretching of the beam. For a slender beam (for example with $\left.I /\left(A l^{2}\right)<0.001\right)$, the linear moment-curvature relationship [26]

$$
\frac{\partial^{2} M}{\partial x^{2}}=E_{0} I v,_{x x x x}+\eta I\left(v,_{x x x x t}+\gamma v,_{x x x x x x}\right)
$$

is sufficiently accurate. Substitution of Eqs (2), (3) and (4) into Eq. (1) yields a non-linear partial-differential equation

$$
\begin{aligned}
& \rho A\left(v,_{t t}+2 \gamma v,_{x t}+\dot{\gamma} v,_{x}+\gamma^{2} v,_{x x}\right)-P_{0} v,_{x x}-\frac{3}{2} E_{0} A v,_{x}^{2} v,_{x x}-2 A \eta v,_{x} v,_{x x} v,_{x t}-A \eta v,_{x}^{2} v,_{x x t} \\
& -2 A \eta \gamma v,_{x} v,_{x}^{2}-A \eta \gamma v,_{x}^{2} v,_{x x x}+E_{0} I v,_{x x x x}+\eta I v,_{x x x x t}+\eta I \gamma v,_{x x x x x}=0 .
\end{aligned}
$$

Equation (5) is the extension of Eq. (10) in [11] to the time-dependent axial speed.

If the spatial variation of the tension is rather small, then one can use the averaged value of the disturbed tension $\frac{1}{l} \int_{0}^{l} P d x$ to replace the exact value $P$. In this case, Eq. (1) becomes

$$
\rho A\left(v, t t+\dot{\gamma} v,_{x}+2 \gamma v,_{x t}+\gamma^{2} v,_{x x}\right)=P_{0} v,_{x x}+\frac{v,,_{x x}}{l} \int_{0}^{l} P d x-\frac{\partial^{2} M}{\partial x^{2}},
$$

which leads to an integro-partial-differential equation

$$
\begin{aligned}
& \rho A\left(v, t t+2 \gamma v,_{x t}+\dot{\gamma} v,_{x}+\gamma^{2} v,_{x x}\right)-P_{0} v,_{x x}-\frac{A v,_{x x}}{l} \int_{0}^{l}\left[\frac{1}{2} E_{0} v,_{x}^{2}+\eta v,_{x} v,_{x t}+\eta \gamma v,_{x} v,_{x x}\right] d x \\
& +E_{0} I v,_{x x x x}+\eta I v,_{x x x x t}+\eta I \gamma v,_{x x x x x}=0 .
\end{aligned}
$$

Consider the axially moving beam constrained by rotating sleeves with rotational springs [31]. The stiffness constant of two springs is the same, denoted as $K$. Nullifying the transverse displacements and balancing the bending moment at both ends lead to the boundary conditions

$$
\begin{gathered}
v(0, t)=0, E_{0} I v,_{x x}(0, t)-K v,_{x}(0, t)=0 ; \\
v(l, t)=0, E_{0} I v,_{x x}(l, t)+K v_{, x}(l, t)=0 .
\end{gathered}
$$

\section{Steady-state response via the method of multiple scales}

Introduce the dimensionless variables and parameters as follows

$$
\begin{aligned}
& v \leftrightarrow \frac{v}{\sqrt{\varepsilon} l}, x \leftrightarrow \frac{x}{l}, t \leftrightarrow t \sqrt{\frac{P_{0}}{\rho A l^{2}}}, \gamma(t) \leftrightarrow \gamma(t) \sqrt{\frac{\rho A}{P_{0}}}, \\
& k_{f}=\sqrt{\frac{E_{0} I}{P_{0} l^{2}}}, \alpha=\frac{I \eta}{\varepsilon L^{3} \sqrt{\rho A P_{0}}}, k_{1}=\sqrt{\frac{E_{0} A}{P_{0} l}}, k_{2}=\frac{\eta \sqrt{A}}{l \varepsilon \sqrt{\rho P_{0}}}, k=\frac{K}{E_{0} I l} .
\end{aligned}
$$

where bookkeeping device $\varepsilon$ is a small dimensionless parameter accounting for the fact that both the transverse displacement and the dynamic viscosity are very small. Equations (5) and (7) can be respectively cast into the dimensionless form

$$
\begin{aligned}
v,_{t t} & +2 \gamma v,_{x t}+\dot{\gamma} v,_{x}+\left(\gamma^{2}-1\right) v,_{x x}+k_{f}^{2} v,_{x x x x}+\varepsilon \alpha v,_{x x x x t}+\varepsilon \alpha \gamma v,_{x x x x x} \\
& =\frac{3}{2} \varepsilon k_{1}^{2} v,_{x}^{2} v,_{x x}+\varepsilon^{2} k_{2} \alpha v,_{x}\left(2 v,_{x x} v,_{x t}+v,_{x} v,_{x x t}+2 \gamma v,_{x x}^{2}+\gamma v,_{x} v,_{x x x}\right), \\
v, t t & +2 \gamma v,_{x t}+\dot{\gamma} v,_{x}+\left(\gamma^{2}-1\right) v,_{x x}+k_{f}^{2} v,_{x x x x}+\varepsilon \alpha v,_{x x x x t}+\varepsilon \alpha \gamma v,_{x x x x x} \\
& =\varepsilon v,{ }_{x x} \int_{0}^{1}\left[\frac{1}{2} k_{1}^{2} v,_{x}^{2}+k_{2} \varepsilon\left(v,_{x} v,_{x t}+\gamma v,_{x} v,_{x x}\right)\right] d x,
\end{aligned}
$$


Boundary conditions Eq. (8) become

$$
v(0, t)=0, v,_{x x}(0, t)-k v,_{x}(0, t)=0 ; v(1, t)=0, v_{, x x}(1, t)+k v,_{x}(1, t)=0 .
$$

In the present investigation, the axial speed is assumed to be a small simple harmonic variation about the constant mean speed,

$$
\gamma(t)=\gamma_{0}+\varepsilon \gamma_{1} \sin \omega t
$$

where $\gamma_{0}$ is the mean axial speed, and $\varepsilon \gamma_{1}$ and $\omega$ are respectively the amplitude and the frequency of the axial speed variation, all in the dimensionless form. Substitution of Eq. (13) into Eqs (10) and (11) respectively yields

$$
\begin{aligned}
& v,_{t t}+2 \gamma_{0} v,_{x t}+\left(\gamma_{0}^{2}-1\right) v,_{x x}+k_{f}^{2} v,_{x x x x}=\varepsilon\left[\frac{3}{2} k_{1}^{2} v,_{x}^{2} v,_{x x}-2 \gamma_{1} \sin \omega t v,_{x t}-2 \gamma_{0} \gamma_{1} \sin \omega t v,_{x x}\right. \\
& \left.-\omega \gamma_{1} \cos \omega t v,_{x}-\alpha v,_{x x x x t}-\alpha \gamma_{0} v,_{x x x x x}\right]+O\left(\varepsilon^{2}\right), \\
& v,_{t t}+2 \gamma_{0} v,_{x t}+\left(\gamma_{0}^{2}-1\right) v,_{x x}+k_{f}^{2} v,_{x x x x}=\varepsilon\left[\frac{1}{2} k_{1}^{2} v,_{x x} \int_{0}^{1} v,_{x}^{2} d x-2 \gamma_{1} \sin \omega t v,_{x t}\right. \\
& \left.-2 \gamma_{0} \gamma_{1} \sin \omega t v,_{x x}-\omega \gamma_{1} \cos \omega t v,_{x}-\alpha v,_{x x x x t}-\alpha \gamma_{0} v,_{x x x x x}\right]+O\left(\varepsilon^{2}\right) .
\end{aligned}
$$

The method of multiple scales can be employed to solve Eqs (14) and (15). Consider Eq. (15) first. A uniform approximation is sought in the form

$$
v(x, t ; \varepsilon)=v_{0}\left(x, T_{0}, T_{1}\right)+\varepsilon v_{1}\left(x, T_{0}, T_{1}\right)+O\left(\varepsilon^{2}\right),
$$

where $T_{0}=t$ is a fast scale characterizing motions occurring at $\omega_{k}$ (one of the natural frequencies of the corresponding unperturbed linear system), and $T_{1}=\varepsilon t$ is a slow scale characterizing the modulation of the amplitudes and phases due to non-linearity, viscoelasticity and possible resonance. Substitution of Eq. (16) and the following relationships

$$
\frac{\partial}{\partial t}=\frac{\partial}{\partial T_{0}}+\varepsilon \frac{\partial}{\partial T_{1}}+O\left(\varepsilon^{2}\right), \frac{\partial^{2}}{\partial t^{2}}=\frac{\partial^{2}}{\partial T_{0}^{2}}+2 \varepsilon \frac{\partial^{2}}{\partial T_{0} \partial T_{1}}+O\left(\varepsilon^{2}\right)
$$

into Eq. (15) and then equalization of coefficients of $\varepsilon^{0}$ and $\varepsilon$ in the resulting equation lead to

$$
\begin{aligned}
v_{0}, T_{0} T_{0} & +2 \gamma_{0} v_{0}, x T_{0}+\left(\gamma_{0}^{2}-1\right) v_{0}, x x+k_{f}^{2} v_{0}, x x x x=0 \\
v_{1}, T_{0} T_{0} & +2 \gamma_{0} v_{1}, x T_{0}+\left(\gamma_{0}^{2}-1\right) v_{1}, x x+k_{f}^{2} v_{1}, x x x x=\frac{1}{2} k_{1}^{2} v_{0}, x_{x x} \int_{0}^{1} v_{0},_{x}^{2} d x-2 v_{0}, T_{0} T_{1}-2 \gamma_{0} v_{0}, x T_{1} \\
& -2 \gamma_{1} \sin \omega T_{0}\left(v_{0}, x T_{0}+\gamma_{0} v_{0},{ }_{x x}\right)-\gamma_{1} \omega \cos \omega T_{0} v_{0},,_{x}-\alpha v_{0}, x x x x T_{0}-\alpha \gamma_{0} v_{0},,_{x x x} .
\end{aligned}
$$

Substitution of Eq. (16) into Eq. (12) yields

$$
\begin{aligned}
& v_{0}(0, t)=0, v_{0},_{x x}(0, t)-k v_{0},{ }_{x}(0, t)=0 ; v_{0}(1, t)=0, v_{0},_{x x}(1, t)+k v_{0},{ }_{x}(1, t)=0, \\
& v_{1}(0, t)=0, v_{1, x x}(0, t)-k v_{1},{ }_{x}(0, t)=0 ; v_{1}(1, t)=0, v_{1,{ }_{x x}}(1, t)+k v_{1},{ }_{x}(1, t)=0 .
\end{aligned}
$$

The modal solution [32] to Eq. (18) is

$$
v_{0}\left(x, T_{0}, T_{1}\right)=\sum_{n=1}^{\infty} \phi_{n}(x) A_{n}\left(T_{1}\right) e^{i \omega_{n} T_{0}}+c c
$$

where $\omega_{n}$ and $\phi_{n}$ are respectively the $n$-th natural frequency and complex mode function of the corresponding linear homogeneous system, and $c c$ denotes the complex conjugate of all preceding terms on the right hand of an equation. Under the boundary condition (20), the modal function corresponding to the $n$-th natural frequency $\omega_{n}$ is [31] 


$$
\begin{aligned}
& \phi_{n}(x)=e^{i \beta_{1 n} x}- \\
& \frac{i k\left(e^{i \beta_{1 n}}+e^{i \beta_{3 n}}\right)\left(\beta_{1 n}-\beta_{3 n}\right)+\left(e^{i \beta_{1 n}}-e^{i \beta_{3 n}}\right)\left[k^{2}+\left(\beta_{1 n}+\beta_{4 n}\right)\left(\beta_{3 n}+\beta_{4 n}\right)\right]}{i k\left(e^{i \beta_{2 n}}+e^{i \beta_{3 n}}\right)\left(\beta_{2 n}-\beta_{3 n}\right)+\left(e^{i \beta_{2 n}}-e^{i \beta_{3 n}}\right)\left[k^{2}+\left(\beta_{2 n}+\beta_{4 n}\right)\left(\beta_{3 n}+\beta_{4 n}\right)\right]} \frac{\left(\beta_{4 n}\right)}{\left(\beta_{2 n}-\beta_{4 n}\right)} e^{i \beta_{2 n} x}- \\
& \frac{i k\left(e^{i \beta_{1 n}}+e^{i \beta_{2 n}}\right)\left(\beta_{1 n}-\beta_{2 n}\right)+\left(e^{i \beta_{1 n}}-e^{i \beta_{2 n}}\right)\left[k^{2}+\left(\beta_{1 n}+\beta_{4 n}\right)\left(\beta_{2 n}+\beta_{4 n}\right)\right]}{i k\left(e^{i \beta_{2 n}}+e^{i \beta_{3 n}}\right)\left(\beta_{2 n}-\beta_{3 n}\right)+\left(e^{i \beta_{2 n}}-e^{i \beta_{3 n}}\right)\left[k^{2}+\left(\beta_{3 n}+\beta_{4 n}\right)\left(\beta_{2 n}+\beta_{4 n}\right)\right]} \frac{\left(\beta_{3 n}-\beta_{4 n}\right)}{\left(i \beta_{3 n} x\right.}- \\
& \left\{1-\frac{i k\left(e^{i \beta_{1 n}}+e^{i \beta_{3 n}}\right)\left(\beta_{1 n}-\beta_{3 n}\right)+\left(e^{i \beta_{1 n}}-e^{i \beta_{3 n}}\right)\left[k^{2}+\left(\beta_{1 n}+\beta_{4 n}\right)\left(\beta_{3 n}+\beta_{4 n}\right)\right]}{i k\left(e^{i \beta_{2 n}}+e^{i \beta_{3 n}}\right)\left(\beta_{2 n}-\beta_{3 n}\right)+\left(e^{i \beta_{2 n}}-e^{i \beta_{3 n}}\right)\left[k^{2}+\left(\beta_{2 n}+\beta_{4 n}\right)\left(\beta_{3 n}+\beta_{4 n}\right)\right]} \frac{\left(\beta_{2 n}-\beta_{4 n}\right)}{-}\right. \\
& \frac{i k\left(e^{i \beta_{1 n}}+e^{i \beta_{2 n}}\right)\left(\beta_{1 n}-\beta_{2 n}\right)+\left(e^{i \beta_{1 n}}-e^{i \beta_{2 n}}\right)\left[k^{2}+\left(\beta_{1 n}+\beta_{4 n}\right)\left(\beta_{2 n}+\beta_{4 n}\right)\right]}{i k\left(e^{i \beta_{2 n}}+e^{i \beta_{3 n}}\right)\left(\beta_{2 n}-\beta_{3 n}\right)+\left(e^{i \beta_{2 n}}-e^{i \beta_{3 n}}\right)\left[k^{2}+\left(\beta_{3 n}+\beta_{4 n}\right)\left(\beta_{2 n}+\beta_{4 n}\right)\right]} \frac{\left(\beta_{3 n}-\beta_{4 n}\right)}{\left(\beta_{3 n} x\right.},
\end{aligned}
$$

where $\beta_{j n}(j=1,2,3,4)$ are four roots of the following 4 th order algebraic equation

$$
k_{f}^{4} \beta_{j n}^{4}+\left(1-\gamma^{2}\right) \beta_{j n}^{2}-2 \omega_{n} \beta_{j n}-\omega_{n}^{2}=0
$$

that can be solved with $\omega_{n}$ from the frequency equation [31]

$$
\begin{aligned}
& e^{i\left(\beta_{1 n}+\beta_{2 n}\right)}\left(\beta_{1 n}-\beta_{2 n}\right)\left(-i k+\beta_{1 n}+\beta_{2 n}\right)\left(\beta_{3 n}-\beta_{4 n}\right)\left(i k+\beta_{3 n}+\beta_{4 n}\right)+e^{i\left(\beta_{1 n}+\beta_{3 n}\right)}\left(\beta_{3 n}-\beta_{1 n}\right) \\
& \left(-i k+\beta_{1 n}+\beta_{3 n}\right)\left(\beta_{2 n}-\beta_{4 n}\right)\left(i k+\beta_{2 n}+\beta_{4 n}\right)+e^{i\left(\beta_{1 n}+\beta_{4 n}\right)}\left(\beta_{2 n}-\beta_{3 n}\right)\left(i k+\beta_{2 n}+\beta_{3 n}\right) \\
& \left(\beta_{1 n}-\beta_{4 n}\right)\left(-i k+\beta_{1 n}+\beta_{4 n}\right)+e^{i\left(\beta_{2 n}+\beta_{3 n}\right)}\left(\beta_{2 n}-\beta_{3 n}\right)\left(-i k+\beta_{2 n}+\beta_{3 n}\right)\left(\beta_{1 n}-\beta_{4 n}\right) \\
& \left(i k+\beta_{1 n}+\beta_{4 n}\right)+e^{i\left(\beta_{2 n}+\beta_{4 n}\right)}\left(\beta_{3 n}-\beta_{1 n}\right)\left(i k+\beta_{3 n}+\beta_{1 n}\right)\left(\beta_{2 n}-\beta_{4 n}\right)\left(-i k+\beta_{2 n}+\beta_{4 n}\right) \\
& +e^{i\left(\beta_{3 n}+\beta_{4 n}\right)}\left(\beta_{1 n}-\beta_{2 n}\right)\left(i k+\beta_{1 n}+\beta_{2 n}\right)\left(\beta_{3 n}-\beta_{4 n}\right)\left(-i k+\beta_{3 n}+\beta_{4 n}\right)=0 .
\end{aligned}
$$

If the speed variation frequency $\omega$ approaches two times that of any natural frequency of the system Eq. (18), principal parametric resonance may occur [14,19,21,22]. A detuning parameter $\sigma$ is introduced to quantify the deviation of $\omega$ from $\omega_{m}$, and $\omega$ is described by

$$
\omega=2 \omega_{m}+\varepsilon \sigma
$$

where $\omega_{m}$ is the $m$-th frequency of free vibration described by Eq. (18). To investigate the principal parametric resonance with the possible contributions of a mode not involved the resonance, the solution to Eq. (18) is expressed as

$$
v_{0}\left(x, T_{0}, T_{1}\right)=\phi_{m}(x) A_{m}\left(T_{1}\right) e^{i \omega_{m} T_{0}}+\phi_{n}(x) A_{n}\left(T_{1}\right) e^{i \omega_{n} T_{0}}+c c
$$

Substituting Eqs (26) and (27) into Eq. (19) and expressing the trigonometric functions in exponential form yield $v_{1}, T_{0} T_{0}+2 \gamma_{0} v_{1}, T_{0}+\left(\gamma_{0}^{2}-1\right) v_{1},{ }_{x x}+k_{f}^{2} v_{1},{ }_{x x x x}$

$$
\begin{aligned}
& =\left[k_{1}^{2}\left|A_{n}\right|^{2} A_{m}\left(\phi_{m}^{\prime \prime} \int_{0}^{1} \overline{\phi^{\prime}}{ }_{n} \phi_{n}^{\prime} d x+\phi_{n}^{\prime \prime} \int_{0}^{1} \overline{\phi^{\prime}}{ }_{n} \phi_{m}^{\prime} d x+\overline{\phi_{n}^{\prime \prime}} \int_{0}^{1} \phi_{n}^{\prime} \phi_{m}^{\prime} d x\right)+\frac{1}{2} k_{1}^{2}\left(\overline{\phi_{m}^{\prime \prime}} \int_{0}^{1} \phi_{m}^{\prime 2} d x+\right.\right. \\
& \left.\left.2 \phi_{m}^{\prime \prime} \int_{0}^{1} \phi_{m}^{\prime} \overline{\phi^{\prime}}{ }_{m} d x\right) A_{m}^{2} \overline{A_{m}}-2 \dot{A}_{m}\left(i \omega_{m} \phi_{m}+\gamma_{0} \phi_{m}^{\prime}\right)+i \gamma_{1} \gamma_{0} \overline{\phi^{\prime \prime}}{ }_{m} \bar{A}_{m} e^{i \sigma T_{1}}-\alpha i \omega_{m} \phi_{m}^{(4)} A_{m}-\alpha \gamma_{0} \phi_{m}^{(5)} A_{m}\right] e^{i \omega_{m} T_{0}} \\
& +\left[k_{1}^{2}\left|A_{m}\right|^{2} A_{n}\left(\phi_{n}^{\prime \prime} \int_{0}^{1} \bar{\phi}_{m}^{\prime} \phi_{m}^{\prime} d x+\phi_{m}^{\prime \prime} \int_{0}^{1} \phi_{n}^{\prime} \bar{\phi}_{m}^{\prime} d x+\bar{\phi}_{m}^{\prime \prime} \int_{0}^{1} \phi_{n}^{\prime} \phi_{m}^{\prime} d x\right)+\frac{1}{2} k_{1}^{2}\left(\overline{\phi_{n}^{\prime \prime}} \int_{0}^{1} \phi_{n}^{\prime 2} d x\right.\right. \\
& \left.+2 \phi_{n}^{\prime \prime} \int_{0}^{1} \phi_{n}^{\prime} \overline{\phi_{n}^{\prime}} d x\right) A_{n}^{2} \overline{A_{n}}-2 \dot{A}_{n}\left(i \omega_{n} \phi_{n}+\gamma_{0} \phi_{n}^{\prime}\right)-\alpha i \omega_{n} \phi_{n}^{(4)} A_{n}-\alpha \gamma_{0} \phi_{n}^{(5)} A_{n} e^{i \omega_{n} T_{0}}+N S T
\end{aligned}
$$


532

L.-Q. Chen et al. / Principal parametric resonance of axially accelerating viscoelastic beams

where the dot and the prime denote derivation with respect to the slow time variable $T_{1}$ and the dimensionless spatial variable $x$ respectively, the over bar denotes complex conjugation, and NST stands for the terms that will not bring secular terms into the solution.

Equation (28) has a bounded solution only if a solvability condition holds. The solvability condition [33] demands that the possible secular term coefficient at the right hand of the Eq. (28) be orthogonal to every solution of the adjoint of the homogeneous problem. That is

$$
\begin{aligned}
& \left\langle k_{1}^{2} A_{m}\left|A_{n}\right|^{2}\left(\phi_{m}^{\prime \prime} \int_{0}^{1} \bar{\phi}_{n}^{\prime} \phi_{n}^{\prime} d x+\overline{\phi_{n}^{\prime \prime}} \int_{0}^{1} \phi_{n}^{\prime} \phi_{m}^{\prime} d x+\phi_{n}^{\prime \prime} \int_{0}^{1} \overline{\phi_{n}^{\prime}} \phi_{m}^{\prime} d x\right)-\alpha i \omega_{m} \phi_{m}^{(4)} A_{m}-\alpha \gamma_{0} \phi_{m}^{(5)} A_{m}+\right. \\
& \left.\frac{1}{2} k_{1}^{2}\left|A_{m}\right|^{2} A_{m}\left(\overline{\phi_{m}^{\prime \prime}} \int_{0}^{1} \phi_{m}^{\prime 2} d x+2 \phi_{m}^{\prime \prime} \int_{0}^{1} \phi_{m}^{\prime} \overline{\phi_{m}^{\prime}} d x\right)-2 \dot{A}_{m}\left(i \omega_{m} \phi_{m}+\gamma_{0} \phi_{m}^{\prime}\right)+i \gamma_{1} \gamma_{0} \bar{\phi}_{m}^{\prime \prime} \bar{A}_{m} e^{i \sigma T_{1}}, \phi_{m}\right\rangle=0 \\
& \left\langle k_{1}^{2}\left|A_{m}\right|^{2} A_{n}\left(\phi_{n}^{\prime \prime} \int_{0}^{1} \bar{\phi}_{m}^{\prime} \phi_{m}^{\prime} d x+\overline{\phi_{m}^{\prime \prime}} \int_{0}^{1} \phi_{n}^{\prime} \phi_{m}^{\prime} d x+\phi_{m}^{\prime \prime} \int_{0}^{1} \phi_{n}^{\prime} \overline{\phi_{m}^{\prime}} d x\right)-\alpha i \omega_{n} \phi_{n}^{(4)} A_{n}-\alpha \gamma_{0} \phi_{n}^{(5)} A_{n}\right. \\
& \left.+\frac{1}{2} k_{1}^{2}\left(\overline{\phi_{n}^{\prime \prime}} \int_{0}^{1} \phi_{n}^{\prime 2} d x+2 \phi_{n}^{\prime \prime} \int_{0}^{1} \phi_{n}^{\prime} \overline{\phi_{n}^{\prime}} d x\right) A_{n}\left|A_{n}\right|^{2}-2 \dot{A}_{n}\left(i \omega_{n} \phi_{n}+\gamma_{0} \phi_{n}^{\prime}\right), \phi_{n}\right\rangle=0
\end{aligned}
$$

where the inner product is defined for complex functions $f(x)$ and $g(x)$ on $[0,1]$ as

$$
\langle f, g\rangle=\int_{0}^{1} f(x) \bar{g}(x) d x .
$$

Application of the distributive law of the inner product to Eqs (29) and (30) respectively leads to

$$
\begin{aligned}
& \dot{A}_{m}+\left(\alpha \mu_{m}-k_{1}^{2} C_{m n}\left|A_{n}\right|^{2}\right) A_{m}+\gamma_{1} e^{i \sigma T_{1}} \chi_{m} \bar{A}_{m}-k_{1}^{2} \kappa_{m} A_{m}^{2} \bar{A}_{m}=0 \\
& \dot{A}_{n}+\left(\alpha \mu_{n}-k_{1}^{2} C_{n m}\left|A_{m}\right|^{2}\right) A_{n}-k_{1}^{2} \kappa_{n} A_{n}^{2} \bar{A}_{n}=0
\end{aligned}
$$

where

$$
\begin{aligned}
\mu_{j}= & \frac{i \omega_{j} \int_{0}^{1} \phi_{j}^{(4)} \bar{\phi}_{j} d x+\gamma_{0} \int_{0}^{1} \phi_{j}^{(5)} \bar{\phi}_{j} d x}{2\left(i \omega_{j} \int_{0}^{1} \phi_{j} \bar{\phi}_{j} d x+\gamma_{0} \int_{0}^{1} \phi_{j}^{\prime} \bar{\phi}_{j} d x\right)}(j=m, n), \\
\chi_{m}= & \frac{i \gamma_{0} \int_{0}^{1} \bar{\phi}_{m}^{\prime \prime} \bar{\phi}_{m} d x}{2\left(i \omega_{m} \int_{0}^{1} \phi_{m} \bar{\phi}_{m} d x+\gamma_{0} \int_{0}^{1} \phi_{m}^{\prime} \bar{\phi}_{m} d x\right)}, \\
C_{m n}= & \frac{\int_{0}^{1} \phi_{m}^{\prime \prime} \bar{\phi}_{m} d x \int_{0}^{1} \bar{\phi}_{n}^{\prime} \phi_{n}^{\prime} d x+\int_{0}^{1} \phi_{n}^{\prime \prime} \bar{\phi}_{m} d x \int_{0}^{1} \bar{\phi}_{n}^{\prime} \phi_{m}^{\prime} d x+\int_{0}^{1} \bar{\phi}_{n}^{\prime \prime} \bar{\phi}_{m} d x \int_{0}^{1} \phi_{n}^{\prime} \phi_{m}^{\prime} d x}{2 \int_{0}^{1}\left(i \omega_{m} \phi_{m}+\gamma_{0} \phi_{m}^{\prime}\right) \bar{\phi}_{m} d x}, \\
C_{n m}= & \frac{1}{2 \int_{0}^{1} \phi_{n}^{\prime \prime} \bar{\phi}_{n} d x \int_{0}^{1} \bar{\phi}_{m}^{\prime} \phi_{m}^{\prime} d x+\int_{0}^{1} \phi_{m}^{\prime \prime} \bar{\phi}_{n} d x \int_{0}^{1} \phi_{n}^{\prime} \bar{\phi}_{m}^{\prime} d x+\int_{0}^{1} \bar{\phi}_{m}^{\prime \prime} \bar{\phi}_{n} d x \int_{0}^{1} \phi_{n}^{\prime} \phi_{m}^{\prime} d x},
\end{aligned}
$$




$$
\kappa_{j}=\frac{\int_{0}^{1} \bar{\phi}_{j} \bar{\phi}^{\prime \prime}{ }_{j} d x \int_{0}^{1}{\phi^{\prime}}_{j}^{2} d x+2 \int_{0}^{1} \phi^{\prime}{ }_{j} \bar{\phi}_{j}^{\prime} d x \int_{0}^{1} \bar{\phi}_{j} \phi_{j}^{\prime \prime} d x}{4\left(i \omega_{j} \int_{0}^{1} \bar{\phi}_{j} \phi_{j} d x+\gamma_{0} \int_{0}^{1} \bar{\phi}_{j} \phi^{\prime}{ }_{j} d x\right)} \quad(j=m, n)
$$

The coefficients $\mu_{j}(j=m, n), \chi_{m}, C_{m n}, C_{n m}$, and $\kappa_{j}(j=m, n)$ are determined by the natural frequencies and the modal function of linear system (18), which are dependent on $\gamma_{0}$ and $k_{f}$, and are independent of $\alpha, \gamma_{1}$ and $k_{1}$. It can be numerically demonstrated that under the given boundary conditions

$$
\operatorname{Re}\left(\mu_{n}\right)>0, \operatorname{Im}\left(\mu_{n}\right)=0 ; \operatorname{Re}\left(C_{j k}\right)=0, \operatorname{Im}\left(C_{j k}\right)>0 ; \operatorname{Re}\left(\kappa_{n}\right)=0, \operatorname{Im}\left(\kappa_{n}\right)<0 .
$$

Express the solution of Eqs (32) and (33) in the polar form

$$
A_{m}\left(T_{1}\right)=a_{m}\left(T_{1}\right) e^{i \varphi_{m}\left(T_{1}\right)}, A_{n}\left(T_{1}\right)=a_{n}\left(T_{1}\right) e^{i \varphi_{n}\left(T_{1}\right)} .
$$

where real functions $a_{m}\left(T_{1}\right)$ and $\varphi_{m}\left(T_{1}\right)$ are respectively the amplitude and the phase angle of the response in the $m$-th principal parametric resonance, and real functions $a_{n}\left(T_{1}\right)$ and $\varphi_{n}\left(T_{1}\right)$ are possible contribution to the response in the $m$-th principal parametric resonance. Substitution of Eq. (38) into Eqs (32) and (33) and separation of real and imaginary parts in the resulting equations give

$$
\begin{aligned}
& \dot{a}_{m}=\left[-\alpha \operatorname{Re}\left(\mu_{m}\right)+\gamma_{1} \operatorname{Im}\left(\chi_{m}\right) \sin \left(\sigma T_{1}-2 \varphi_{m}\right)-\gamma_{1} \operatorname{Re}\left(\chi_{m}\right) \cos \left(\sigma T_{1}-2 \varphi_{m}\right)\right] a_{m}, \\
& a_{m} \dot{\varphi}_{m}=-\gamma_{1}\left[\operatorname{Re}\left(\chi_{m}\right) \sin \left(\sigma T_{1}-2 \varphi_{m}\right)+\operatorname{Im}\left(\chi_{m}\right) \cos \left(\sigma T_{1}-2 \varphi_{m}\right)\right] a_{m} \\
& \quad-k_{1}^{2} \operatorname{Im}\left(C_{m n}\right) a_{n}^{2} a_{m}+k_{1}^{2} \operatorname{Im}\left(\kappa_{m}\right) a_{m}^{3} \\
& \dot{a}_{n}=-\alpha \operatorname{Re}\left(\mu_{n}\right) a_{n}, \\
& a_{n} \dot{\varphi}_{n}=-k_{1}^{2} \operatorname{Im}\left(C_{n m}\right)\left|A_{m}\right|^{2} a_{n}+k_{1}^{2} \operatorname{Im}\left(\kappa_{n}\right) a_{n}^{3},
\end{aligned}
$$

From Eq. (40), $a_{n}$ decays to zero exponentially. Therefore, the $n$-th mode has actually no effect on the $m$-th principal parametric resonance.

Inserting $a_{n}=0$ into Eq. (39) leads to

$$
\begin{aligned}
& \dot{a}_{m}=\left[-\alpha \operatorname{Re}\left(\mu_{m}\right)+\gamma_{1} \operatorname{Im}\left(\chi_{m}\right) \sin \theta_{m}-\gamma_{1} \operatorname{Re}\left(\chi_{m}\right) \cos \theta_{m}\right] a_{m}, \\
& a_{m} \dot{\theta}_{m}=\sigma a_{m}+2 \gamma_{1}\left[\operatorname{Re}\left(\chi_{m}\right) \sin \theta_{m}+\operatorname{Im}\left(\chi_{m}\right) \cos \theta_{m}\right] a_{m}-2 k_{1}^{2} \operatorname{Im}\left(\kappa_{m}\right) a_{m}^{3},
\end{aligned}
$$

where

$$
\theta_{m}=\sigma T_{1}-2 \varphi_{m}
$$

For the steady-state response, the amplitude $a_{m}$ and the new phase angle $\theta_{m}$ in Eq. (41) are constant [31,33]. Locating the fixed points, one can find the amplitudes of the steady-state responses. There is a fixed point at the origin (trivial zero solution), which represents the straight equilibrium configuration of the beam. In addition, there may exist nontrivial periodic solution with amplitudes defined by

$$
\begin{aligned}
& a_{m 1}=\frac{\sqrt{\operatorname{Im}\left(\kappa_{m}\right)}}{k_{1}} \sqrt{2 \sigma+4 \sqrt{\gamma_{1}^{2}\left|\chi_{m}\right|^{2}-\alpha^{2}\left[\operatorname{Re}\left(\mu_{m}\right)\right]^{2}}}, \\
& a_{m 2}=\frac{\sqrt{I m\left(\kappa_{m}\right)}}{k_{1}} \sqrt{2 \sigma-4 \sqrt{\gamma_{1}^{2}\left|\chi_{m}\right|^{2}-\alpha^{2}\left[\operatorname{Re}\left(\mu_{m}\right)\right]^{2}}} .
\end{aligned}
$$

From Eqs (43) and (44), it can be concluded that the nontrivial steady-state solutions exist only if the following conditions hold,

$$
\alpha \leqslant \frac{\gamma_{1}\left|\chi_{m}\right|}{R e\left(\mu_{m}\right)}
$$

for both nontrivial solutions, 


$$
\sigma \geqslant \sigma_{1}=-2 \sqrt{\gamma_{1}^{2}\left|\chi_{m}\right|^{2}-\alpha^{2}\left[\operatorname{Re}\left(\mu_{m}\right)\right]^{2}}
$$

for solution (43), and

$$
\sigma \geqslant \sigma_{2}=2 \sqrt{\gamma_{1}^{2}\left|\chi_{m}\right|^{2}-\alpha^{2}\left[\operatorname{Re}\left(\mu_{m}\right)\right]^{2}}
$$

for solution (44), It can be proved [14] that the zero solution is unstable for $\sigma \in\left[\sigma_{1}, \sigma_{2}\right]$, solution (43) is always stable, and solution (44) is always unstable.

From Eqs (16), (26), (27) and (38), the approximate expression of the stable steady-state responses in the $m$-th principal parametric resonance is

$$
u_{0}(x, t)=2 a_{m 1}\left|\phi_{m}(x)\right| \cos \left(\frac{\omega}{2} t-\frac{1}{2} \theta_{m 1}+\arccos \frac{R e \phi_{m}(x)}{\left|\phi_{m}(x)\right|}\right),
$$

where

$$
\theta_{m 1}=\frac{1}{2} \arcsin \left(1-\frac{\alpha^{2} R e^{2}\left(\mu_{m}\right)}{\gamma_{1}^{2}\left|\chi_{m}\right|^{2}}\right)
$$

Hence the amplitude of the stable steady-state response is

$$
A(x)=2 a_{m 1}\left|\phi_{m}(x)\right| \text {. }
$$

Principal parametric resonance in the system governed by Eq. (14) can be analyzed via the method of multiple scales in a similar way. The solvability condition is still expressed by Eqs (32) and (33) with coefficients given by Eq. (34), while the other coefficients are defined by the following equations rather than Eqs (35) and (36)

$$
\begin{aligned}
& C_{n m}= \frac{3 \int_{0}^{1}\left(\phi_{n}^{\prime} \bar{\phi}_{m}^{\prime} \phi_{m}^{\prime \prime}+\phi_{n}^{\prime} \phi_{m}^{\prime} \bar{\phi}_{m}^{\prime \prime}+\phi_{m}^{\prime} \bar{\phi}_{m}^{\prime} \phi_{n}^{\prime \prime}\right) \bar{\phi}_{n} d x}{2 \int_{0}^{1}\left(i \omega_{n} \phi_{n}+\gamma_{0} \phi_{n}^{\prime}\right) \bar{\phi}_{n} d x} \\
& C_{m n}=\frac{3 \int_{0}^{1}\left(\phi_{m}^{\prime} \bar{\phi}_{n}^{\prime} \phi_{n}^{\prime \prime}+\phi_{m}^{\prime} \phi_{n}^{\prime} \bar{\phi}_{n}^{\prime \prime}+\phi_{n}^{\prime} \bar{\phi}_{n}^{\prime} \phi_{m}^{\prime \prime}\right) \bar{\phi}_{m} d x}{2 \int_{0}^{1}\left(i \omega_{m} \phi_{m}+\gamma_{0} \phi_{m}^{\prime}\right) \bar{\phi}_{m} d x} \\
& \kappa_{k}=\frac{3\left(\int_{0}^{1} \bar{\phi}_{k} \bar{\phi}^{\prime \prime}{ }_{k} \phi^{\prime 2}{ }_{k} d x+\frac{3}{2} \int_{0}^{1} \bar{\phi}_{k} \phi^{\prime \prime}{ }_{k} \phi^{\prime}{ }_{k} \bar{\phi}_{k}^{\prime} d x\right)}{4\left(i \omega_{k} \int_{0}^{1} \phi_{k} \bar{\phi}_{k} d x+\gamma_{0} \int_{0}^{1} \phi_{k}{ }_{k} \bar{\phi}_{k} d x\right)}
\end{aligned}
$$

\section{Numerical illustrations of the analytical results}

The effects of some parameters on the amplitude and the existence conditions can be inferred from Eqs (43), (44), (46), and (47). From Eq. (43), $a_{n 1}$ increases with $\sigma$ and $\gamma_{1}$, and decreases with $k_{1}$ and $\alpha$. From Eq. (46), $a_{n 2}$ increases with $\sigma$ and $\alpha$, and decreases with $k_{1}$ and $\gamma_{1}$. From Eq. (46), $\sigma_{1}$ increases with $\alpha$, and decreases $\gamma_{1}$. From Eq. (46), $\sigma_{1}$ increases with $\gamma_{1}$, and decreases $\alpha$. That is, $\gamma_{1}$ makes the instability interval of the straight equilibrium configuration smaller, and $\alpha$ makes it larger. However, to understand the effects of other parameters such as $n, \gamma_{0}$, and $k$, numerical examples are necessary.

Consider an axially moving beam with $k_{f}=0.8$. For given $\gamma_{0}$ and $k$, the modal parameters can be numerically solved from Eqs (24) and (25), and coefficients defined by Eqs (34)-(36), (51) and (52) can be calculated. For example, let $\gamma_{0}=2.0$, and $k=0$. Then $\omega_{1}=5.3692$ and $\omega_{2}=30.1200$, and $\beta_{j n}(j=1,2,3,4)$ and the coefficients $\mu_{j}(j=m, n), \chi_{m}, C_{m n}, C_{n m}$, and $\kappa_{j}(j=m, n)$ are calculated for Eqs (24) and (25). The numerical result is 
Table 1

The roots of Eq. (24) and $\mu_{n}, \chi_{n}$ calculated from Eq. (34)

\begin{tabular}{ccccccc}
\hline$n$ & $\beta_{1 n}$ & $\beta_{2 n}$ & $\beta_{3 n}$ & $\beta_{4 n}$ & $\mu_{n}$ & $\chi_{n}$ \\
\hline 1 & 4.57 & $-1.25+3.07 \mathrm{i}$ & $-1.25-3.07 \mathrm{i}$ & -2.07 & 148.57 & $-0.15+1.03 \mathrm{i}$ \\
2 & 7.73 & $-1.25+6.36 \mathrm{i}$ & $-1.25-6.36 \mathrm{i}$ & -5.23 & 1145.52 & $-0.12+0.97 \mathrm{i}$ \\
\hline
\end{tabular}

Table 2

The coefficients respectively calculated from Eqs (35), (36) and (51), (52)

\begin{tabular}{ccccc}
\hline & $C_{12}$ & $C_{21}$ & $\kappa_{1}$ & $\kappa_{2}$ \\
\hline Eq. (15) & $170.509 \mathrm{i}$ & $56.6002 \mathrm{i}$ & $-40.2494 \mathrm{i}$ & $-142.864 \mathrm{i}$ \\
Eq. (14) & $69.4427 \mathrm{i}$ & $23.0514 \mathrm{i}$ & $-28.8418 \mathrm{i}$ & $-88.5134 \mathrm{i}$ \\
\hline
\end{tabular}

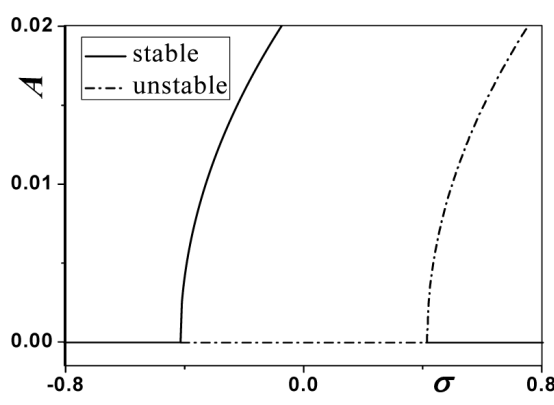

(a) $m=1$

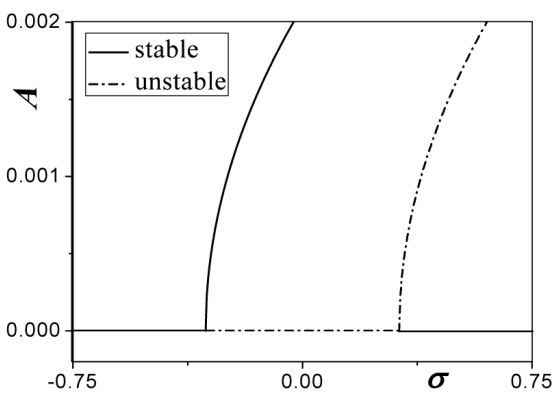

(b) $m=2$

Fig. 1. Amplitudes and stability of steady-state responses: Eq. (15).

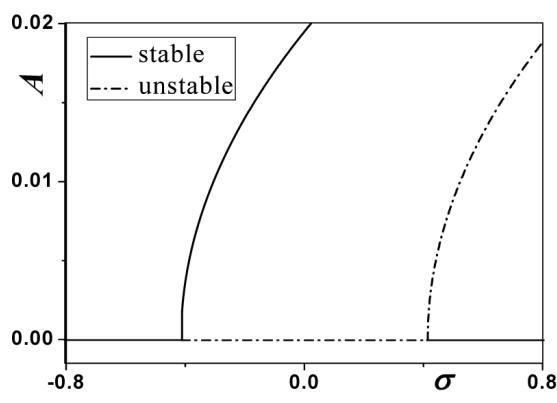

(a) $m=1$

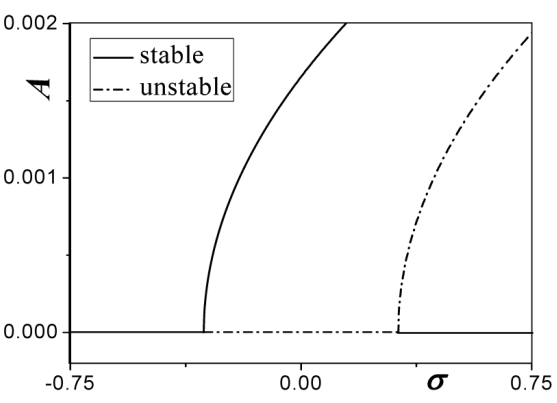

(b) $m=2$

Fig. 2. Amplitudes and stability of steady-state responses: Eq. (14).

shown in Tables 1 and 2. Similarly, let $\gamma_{0}=2.0$, and $k=2.0$. Then $\omega_{1}=8.1570, \omega_{2}=32.9441$ and the coefficients can be obtained but are not listed.

Figures 1 and 2 illustrate the relationship between the amplitude at the beam center and the detuning parameter for first two principal parametric resonances for Eqs (14) and (15) respectively. In the both figures, the solid or dot lines stand for stable or unstable amplitudes respectively. Equations (14) and (15) yield the qualitatively same results. In both the first and the second principal resonance, only the trivial zero solution exists and is stable for $\sigma<\sigma_{1}$. At $\sigma=\sigma_{1}$, the trivial solution losses its stability and a stable nontrivial solution occurs. At $\sigma=\sigma_{2}$, the unstable trivial solution becomes stable again, and an unstable nontrivial solution bifurcates. The response amplitude of the first principal resonance is much larger than that of the second principal resonance, and the instability interval in the first principal resonance is larger than that in the second principal resonance. Therefore, the low order principal resonance is more significant.

The effects of the mean axial speed are shown in Figs 3 and 4 with $k_{f}=0.8, k_{1}=20, \alpha=0.0001, k=2.0$, and $\gamma_{1}=0.2$. Eqs (14) and (15) predict the same tendencies. The instability interval increases with the mean speed. 


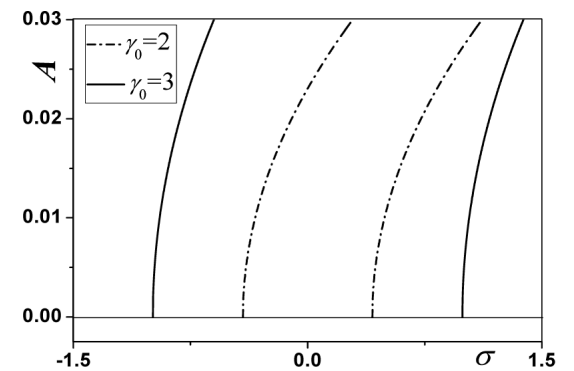

(a) $m=1$

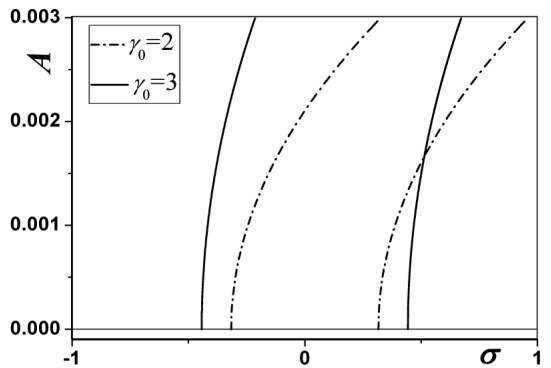

(b) $m=2$

Fig. 3. Effects of the mean axial speed: Eq. (15).

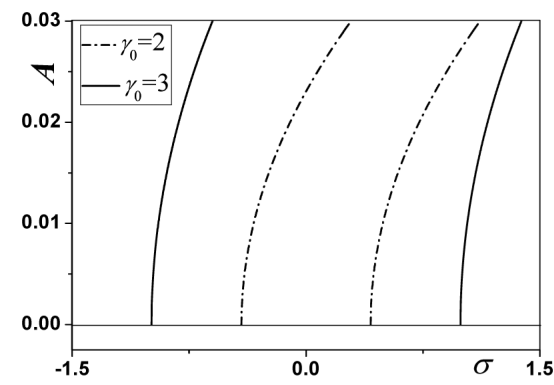

(a) $m=1$

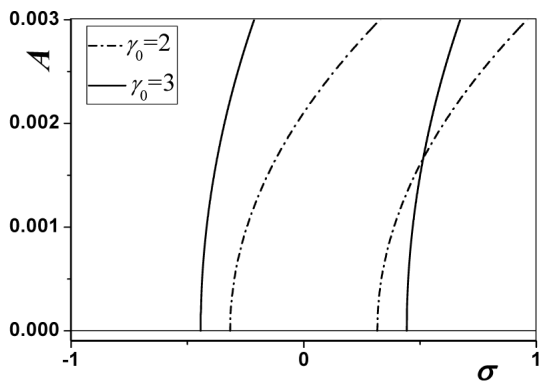

(b) $m=2$

Fig. 4. Effects of the mean axial speed: Eq. (14).

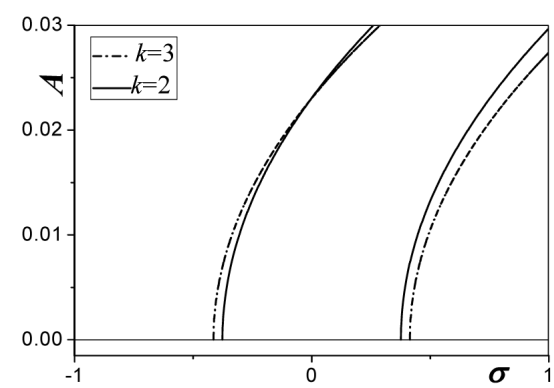

(a) $m=1$

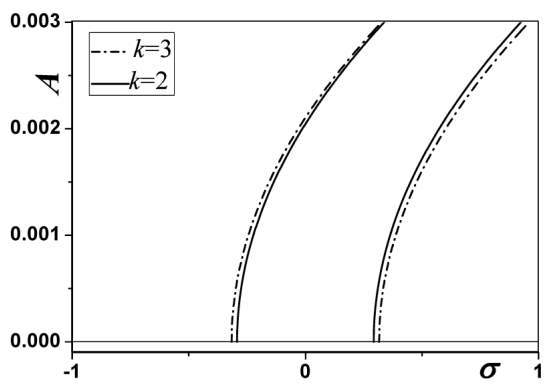

(b) $m=2$

Fig. 5. Effects of the rotational spring stiffness: Eq. (15).

The effects of the rotational spring stiffness are shown in Figs 5 and 6 with $k_{f}=0.8, k_{1}=20, \alpha=0.0001, \gamma_{0}=$ 2.0, and $\gamma_{1}=0.2$. Equations (14) and (15) predict the same tendencies again. The instability interval decreases with the spring stiffness.

The numerical examples indicate that results of Eqs (14) and (15) are qualitatively same, while there exist quantitative differences. Figure 7 is the superposition of Figs 1 and 2. The amplitude derived from Eq. (14) is smaller, and the instability intervals are the same.

\section{Stable steady-state response via the differential quadrature method}

Stable steady-state response can be determined by solving the governing equations numerically. In the following, the differential quadrature method [24,25,27,28] is applied to calculate stable steady-state responses. In numerical investigations here, let $\varepsilon=1$ in Eqs (10) and (11). 


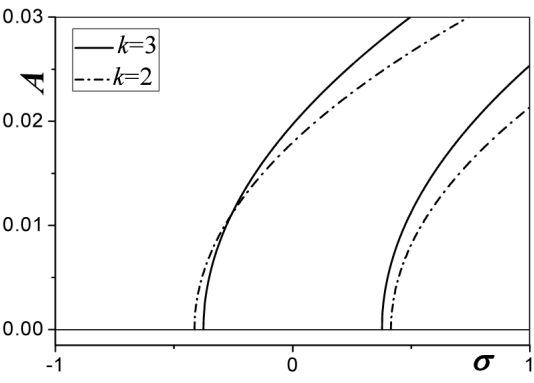

(a) $m=1$

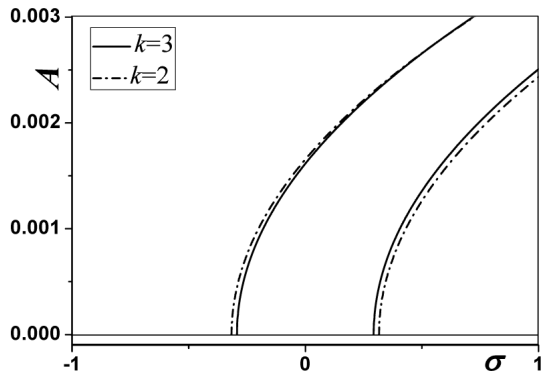

(b) $m=2$

Fig. 6. Effects of the rotational spring stiffness: Eq. (14).

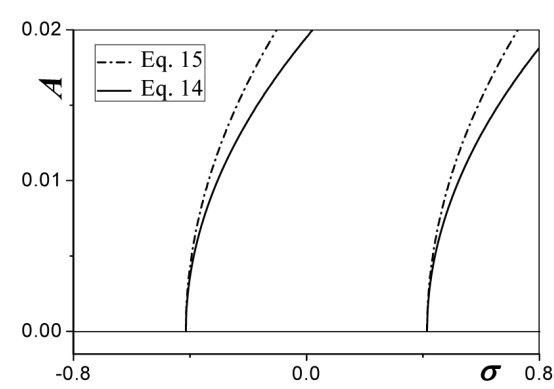

(a) $m=1$

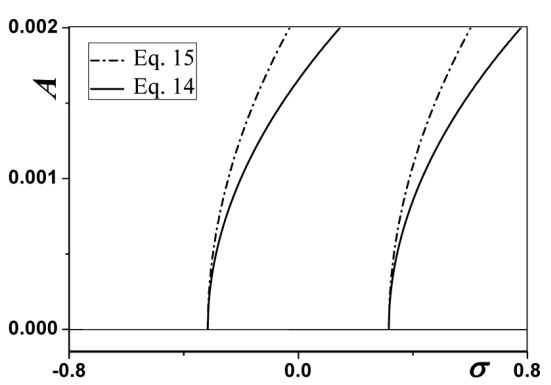

(b) $m=2$

Fig. 7. Comparison of results from Eqs (14) and (15).

Introduce $N$ unequally spaced sampling points as

$$
x_{i}=\frac{1}{2}\left[1-\cos \frac{(i-1) \pi}{N-1}\right] \quad(i=1,2, \ldots, N) .
$$

The quadrature rules for the derivatives of a function at the sampling points yield

$$
\begin{aligned}
& v,_{x}\left(x_{i}, t\right)=\sum_{j=1}^{N} A_{i j}^{(1)} v\left(x_{j}, t\right), v,_{x x}\left(x_{i}, t\right)=\sum_{j=1}^{N} A_{i j}^{(2)} v\left(x_{j}, t\right), \\
& v,_{x x x x}\left(x_{i}, t\right)=\sum_{j=1}^{N} A_{i j}^{(4)} v\left(x_{j}, t\right), v,_{x x x x x}\left(x_{i}, t\right)=\sum_{j=1}^{N} A_{i j}^{(5)} v\left(x_{j}, t\right),
\end{aligned}
$$

where the weighting coefficients are the expression [27]

$$
A_{i j}^{(1)}=\frac{\prod_{k=1, k \neq i}^{N}\left(x_{i}-x_{k}\right)}{\left(x_{i}-x_{j}\right) \prod_{k=1, k \neq j}^{N}\left(x_{j}-x_{k}\right)}(i, j=1,2, \cdots, N ; j \neq i)
$$

and the recurrence relationship

$$
\begin{aligned}
& A_{i j}^{(r)}=r\left[A_{i i}^{(r-1)} A_{i j}^{(1)}-\frac{A_{i j}^{(r-1)}}{x_{i}-x_{j}}\right] \quad(r=2,3,4,5 ; i, j=1,2, \cdots, N ; j \neq i), \\
& A_{i i}^{(r)}=-\sum_{k=1, k \neq i}^{N} A_{i k}^{(r)}(r=1,2,3,4,5 ; i=1,2, \cdots, N) .
\end{aligned}
$$


Consider the beam simply supported at both ends. Then the boundary conditions are

$$
v(0, t)=0, v,_{x x}(0, t)=0, v(1, t)=0, v,_{x x}(1, t)=0 .
$$

Substitution of Eq. (54) into Eqs (10) and (58) and modification of the weighting coefficient matrices to implement the boundary conditions [34] yield

$$
\begin{aligned}
& \ddot{v}_{j}+\left\{-k_{2}\left(\sum_{k=1}^{N} A_{j k}^{(1)} v_{k}\right)\left\{2\left[\sum_{k=1}^{N} A_{j k}^{(2)} v_{k}\right] \sum_{k=1}^{N} A_{j k}^{(1)}+\left[\sum_{k=1}^{N} A_{j k}^{(1)} v_{k}\right] \sum_{k=1}^{N} A_{j k}^{(2)}\right\}\right. \\
& \left.+\sum_{k=1}^{N}\left[2 \gamma A_{j k}^{(1)}+\alpha A_{j k}^{(4)}\right]\right\} \dot{v}_{k}-\sum_{k=1}^{N}\left[\dot{\gamma} A_{j k}^{(1)}+\left(\gamma^{2}-1\right) A_{j k}^{(2)}+k_{f}^{2} A_{j k}^{(4)}+\alpha \gamma A_{j k}^{(5)}\right] v_{k} \\
& =\gamma k_{2}\left[\sum_{k=1}^{N} A_{j k}^{(1)} v_{k}\right]\left\{2\left[\sum_{k=1}^{N} A_{j k}^{(2)} v_{k}\right]^{2}+\left[\sum_{k=1}^{N} A_{j k}^{(1)} v_{k}\right]\left[\sum_{k=1}^{N} A_{j k}^{(3)} v_{k}\right]\right\} \\
& +\frac{3}{2} k_{1}^{2}\left(\sum_{k=1}^{N} A_{j k}^{(2)} v_{k}\right)\left(\sum_{k=1}^{N} A_{j k}^{(1)} v_{k}\right)^{2}, \quad(j=2,3,4, \ldots, N-1), \quad v_{0}=v_{N}=0
\end{aligned}
$$

where

$$
v_{j}(t)=v\left(x_{j}, t\right) \quad(j=1,2, \ldots, N) .
$$

Similarly, Eq. (11) under boundary condition (58) can be discretized into

$$
\begin{aligned}
& \ddot{v}_{j}+\left(\sum_{k=1}^{N}\left(2 \gamma A_{j k}^{(1)}+\alpha A_{j k}^{(4)}\right)\right) \dot{v}_{k}+\sum_{k=1}^{N}\left[\dot{\gamma} A_{j k}^{(1)}+\left(\gamma^{2}-1\right) A_{j k}^{(2)}+v_{f}^{2} A_{j k}^{(4)}+\alpha \gamma A_{j k}^{(5)}\right] v_{k} \\
& =\left(\sum_{k=1}^{N} A_{j k}^{(2)} v_{k}\right)\left\{\sum _ { g = 1 } ^ { N } I _ { g } \left[\frac{1}{2} k_{1}^{2}\left(\sum_{k=1}^{N} A_{g k}^{(1)} v_{k}\right)^{2}+\gamma k_{2}\left(\sum_{k=1}^{N} A_{g k}^{(2)} v_{k}\right)\left(\sum_{k=1}^{N} A_{g k}^{(1)} v_{k}\right)\right.\right. \\
& \left.\left.+k_{2}\left(\sum_{k=1}^{N} A_{g k}^{(1)} v_{k}\right)\left(\sum_{k=1}^{N} A_{g k}^{(1)} \dot{v}_{k}\right)\right]\right\}, \quad(j=2,3, \ldots, N-1), \quad v_{0}=v_{N}=0,
\end{aligned}
$$

where weighting coefficients $I_{g}(g=1,2, \ldots, N)$ for integrals are solved from

$$
\left(\begin{array}{ccccc}
1 & 1 & \cdots & 1 & 1 \\
x_{1} & x_{2} & \cdots & x_{N-1} & x_{N} \\
\vdots & \vdots & & \vdots & \vdots \\
x_{1}^{N-2} & x_{2}^{N-2} & \cdots & x_{N-2}^{N-2} & x_{N}^{N-2} \\
x_{1}^{N-1} & x_{1}^{N-1} & \cdots & x_{N-1}^{N-1} & x_{N}^{N-1}
\end{array}\right)\left(\begin{array}{c}
I_{1} \\
I_{2} \\
\vdots \\
I_{N-1} \\
I_{N}
\end{array}\right)=\left(\begin{array}{c}
1 \\
1 / 2 \\
\vdots \\
1 /(N-1) \\
1 / N
\end{array}\right) .
$$

For a set of given parameters $k_{f}, \gamma_{0}, \gamma_{1}, \omega, \alpha, k_{1}$, and $k_{2}, v_{j}$ can be numerically solved from Eq. (59) or (61). For an odd $N, v_{(N+1) / 2}$ is the beam center displacement. The stable steady-state response has constant local maximums of $v_{(N+1) / 2}$, which is the amplitude of the beam center $A$. Changing $\omega$ and calculating corresponding $A$ yield the curve of the amplitude via the detuning parameter.

Consider an axially moving beam with $k_{f}=0.8$ and $\gamma_{0}=2.0$. Then the first two natural frequencies of system (18) are $\omega_{1}=5.3692$ and $\omega_{2}=30.12$. Let $\gamma_{1}=0.2, \alpha=0.0001$, and $k_{1}=20$. Based on the numerical solutions of Eq. (61), Fig. 8 shows the amplitudes of the stable steady-state responses changing with the detuning parameter. The numerical results demonstrate that the lower order principal resonance has the larger stable response amplitude and the larger instability interval of the straight equilibrium.

Figure 9 shows the effects of the mean axial speed based on the numerical solutions of Eq. (61). In Fig. 9, $k_{f}=$ $0.8, k_{1}=20, k_{2}=0, \alpha=0.0001$, and $\gamma_{1}=0.2$. The larger mean axial speed leads to the larger stable response amplitude and the larger instability interval of the straight equilibrium. 


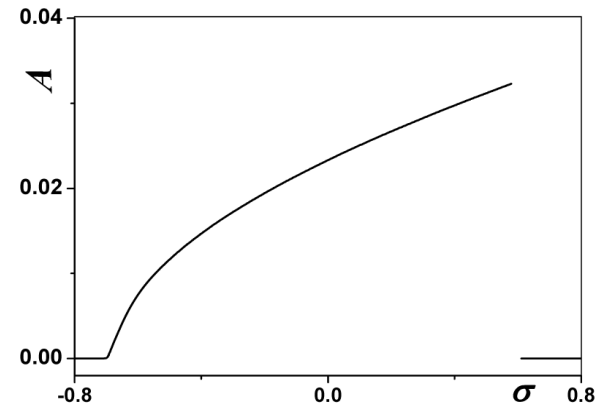

(a) $m=1$

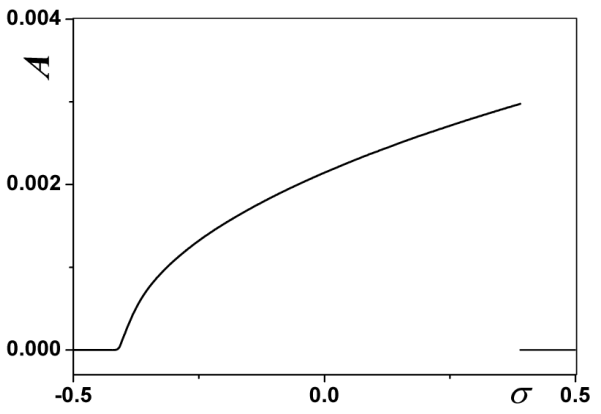

(b) $m=2$

Fig. 8. Amplitudes of stable steady-state responses.

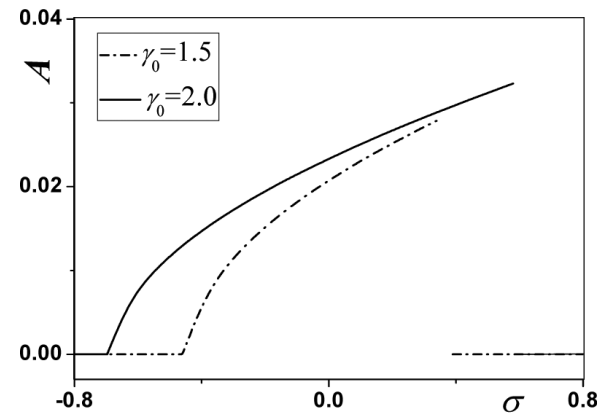

(a) $m=1$

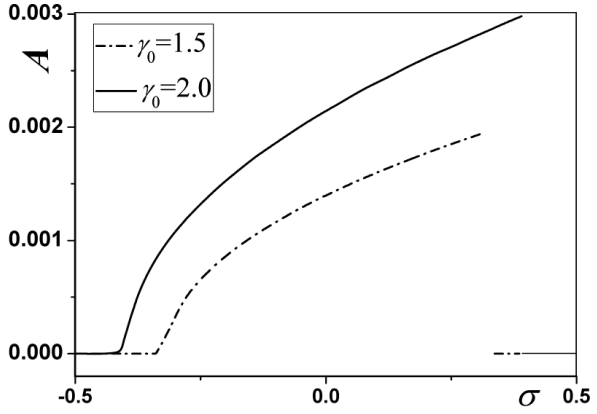

(b) $m=2$

Fig. 9. Effects of the mean axial speed.

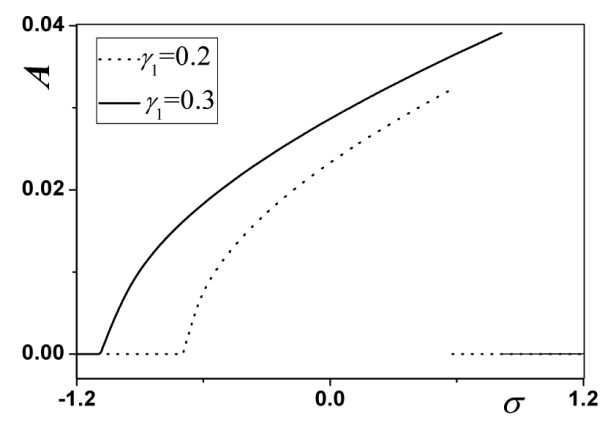

(a) $m=1$

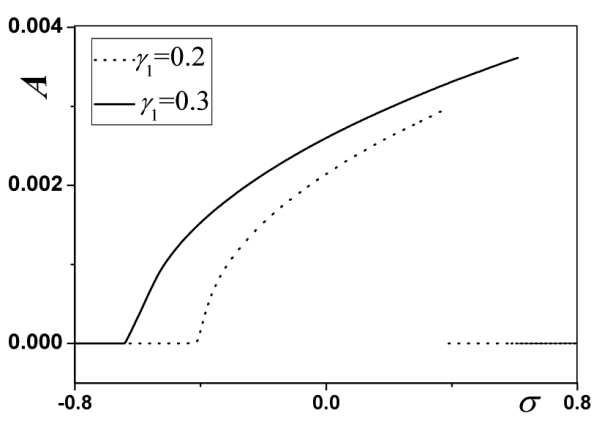

(b) $m=2$

Fig. 10. Effects of the axial speed variation amplitude.

Figure 10 shows the effects of the axial speed variation amplitude based on the numerical solutions of Eq. (61). In Fig. $10, k_{f}=0.8, k_{1}=20, k_{2}=0, \alpha=0.0001$, and $\gamma_{0}=2.0$. Both the stable response amplitude and the equilibrium instability interval increase with the axial speed variation amplitude.

Figure 11 shows the effects of the dynamic viscosity based on the numerical solutions of Eq. (61). In Fig. 11, $k_{f}=0.8, k_{1}=20, k_{2}=0, \gamma_{1}=0.2$, and $\gamma_{0}=2.0$. The larger dynamic viscosity leads to the smaller stable response amplitude and the smaller instability interval of the straight equilibrium.

Figure 12 shows the effects of the non-linear coefficient $k_{1}$ based on the numerical solutions of Eq. (61). In Fig. $12, k_{f}=0.8, k_{2}=0, \alpha=0.0001, \gamma_{0}=2.0$, and $\gamma_{1}=0.2$. The stable response amplitude decreases with the non-linear coefficient $k_{1}$, while the equilibrium instability interval is independent of $k_{1}$. 


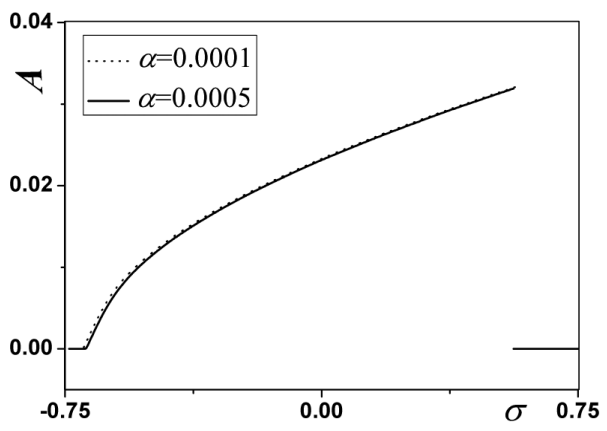

(a) $m=1$

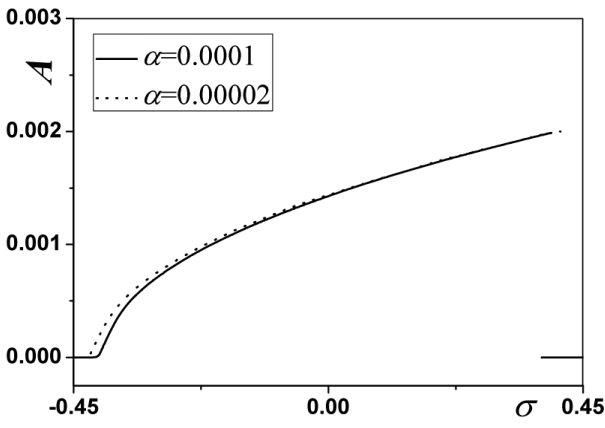

(b) $m=2$

Fig. 11. Effects of the dynamic viscosity.

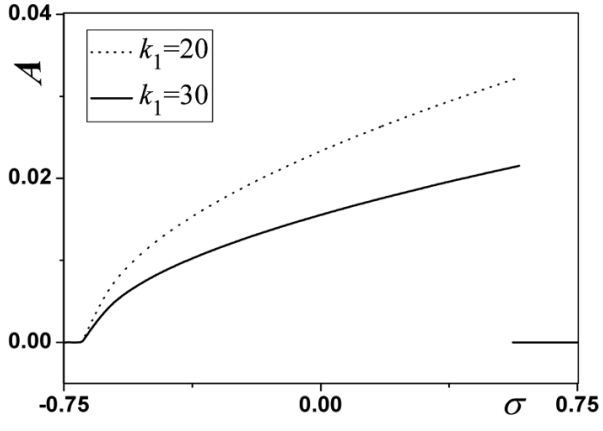

(a) $m=1$

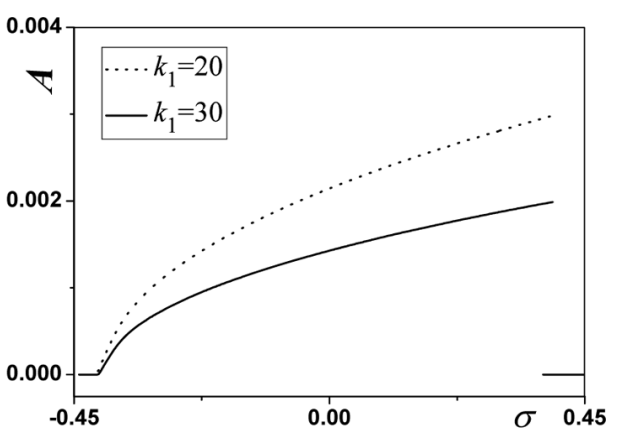

(b) $m=2$

Fig. 12. Effects of the non-linear coefficient $k_{1}$.

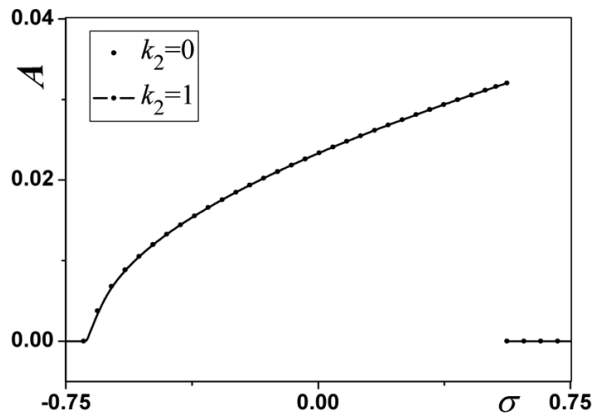

(a) $m=1$

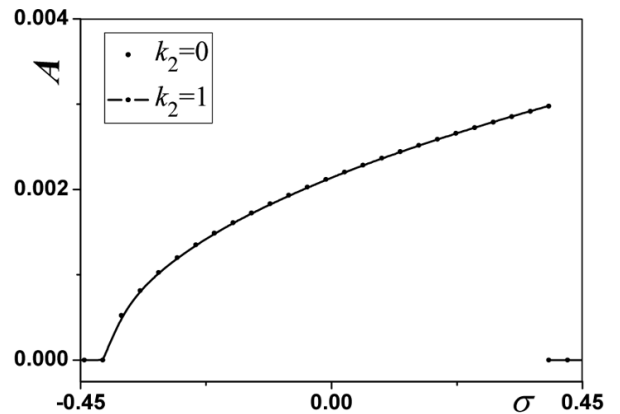

(b) $m=2$

Fig. 13. Effects of the non-linear coefficient $k_{2}$.

Figure 13 shows the effects of the non-linear coefficient $k_{2}$ based on the numerical solutions of Eq. (61). In Fig. $13, k_{f}=0.8, k_{1}=20, \alpha=0.0001, \gamma_{0}=2.0$, and $\gamma_{1}=0.2$. In practical cases, $k_{2}$ is usual smaller than 1 . The stable response amplitude and the equilibrium instability interval are not sensitive to $k_{2}$.

Equation (59) yields the qualitatively same changing tendencies as Eq. (61), so that the numerical results are not shown. The quantitative comparisons between Eqs (59) and (61) are made in Fig. 14. In Fig. 14, $k_{f}=0.8, k_{1}=20$, $k_{2}=0, \alpha=0.0001, \gamma_{0}=2.0, \gamma_{1}=0.2$. The amplitude based on the numerical solution of Eq. (59) is smaller, and the instability intervals are the same. 


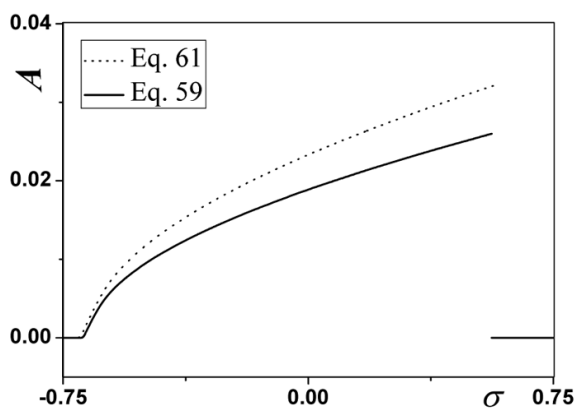

(a) $m=1$

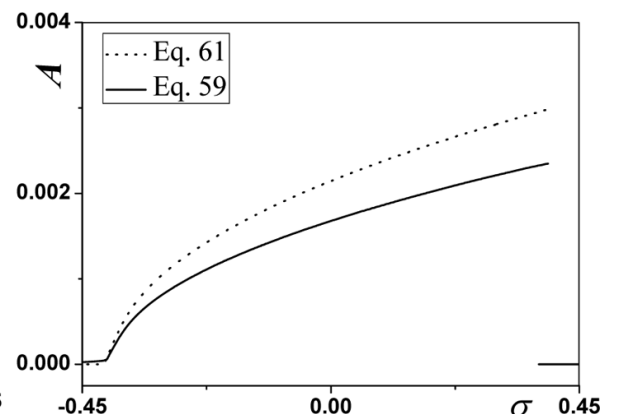

(b) $m=2$

Fig. 14. Comparison of results from Eqs (59) and (61).

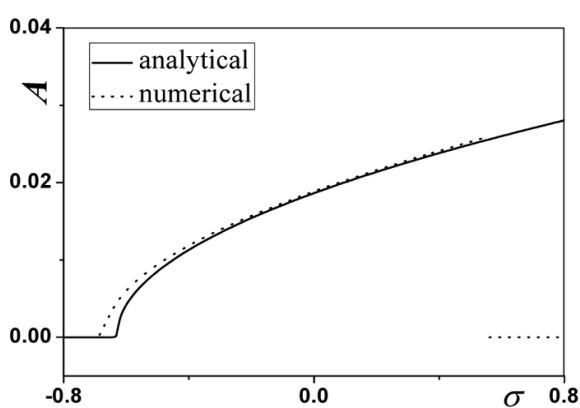

(a) $m=1$

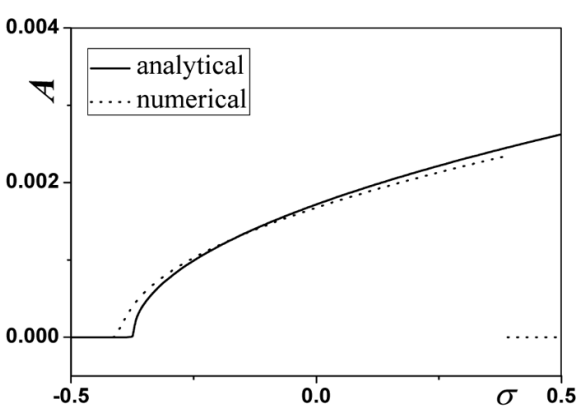

(b) $m=2$

Fig. 15. Comparison of analytical and numerical results: Eq. (10).

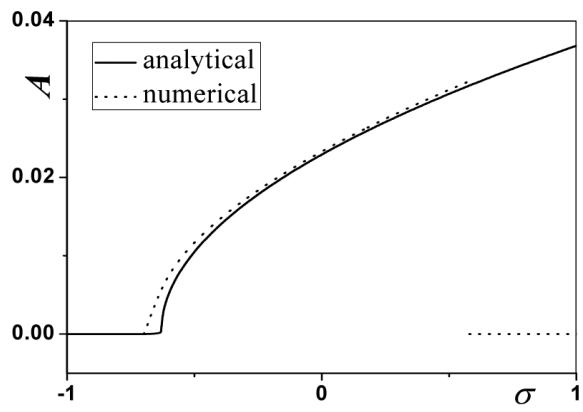

(a) $m=1$

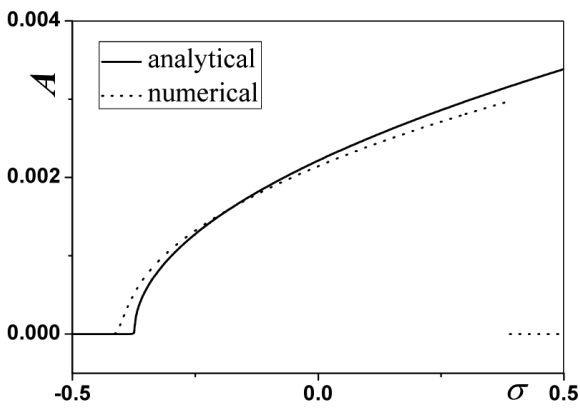

(b) $m=2$

Fig. 16. Comparison of analytical and numerical results: Eq. (11).

\section{Comparisons of approximate analytical results and the numerical results}

The stable steady-state response can be determined via the method of multiple scales, as documented in Sec. 3, and calculated via the differential quadrature method, as presented in Sec. 5. Although the analytical studies considered a more general boundary conditions, the boundary conditions can easily reduced to the simple supports by inserting $K=0$ into Eq. (8). The both results are quantitatively contrasted in the case with $k_{f}=0.8, k_{1}=$ $20, k_{2}=0, \alpha=0.0001, \gamma_{0}=2.0, \gamma_{1}=0.2$. Figures 15 and 16 show the comparison. The amplitudes from both methods are almost coincided, especially near the exact-resonance $(\sigma=0)$ and in the first resonance. However, there are small errors in the equilibrium instability interval, especially for Eq. (10). On the whole, it can be concluded that the outcomes of the multi-scale method are verified by the differential quadrature method. 


\section{Conclusions}

This paper is devoted to steady-state response in principal parametric resonance of axially accelerating viscoelastic beams. The method of multiple scales is applied to a non-linear partial-differential equation and a non-linear integropartial-differential equation to determine steady-state responses. The differential quadrature schemes are developed to verify the approximate analytical results. The investigation leads to the following conclusions.

(1) Analytical study demonstrates that the mode uninvolved in the resonance has no effect on the steady-state response.

(2) Both analytical study and numerical calculations demonstrate that, when the axial speed variation frequency is close to 2 times of any linear natural frequency enough, or the detuning parameter belongs to an interval, the straight equilibrium configuration become unstable. The instability interval increases with the mean and the variation amplitude of the axial speed, decreases with the principal parametric resonance order and the dynamic viscosity, and is independent of non-linear coefficients. Analytical study also demonstrates that the instability interval decreases with the boundary constraint stiffness.

(3) Both analytical study and numerical calculations demonstrate that, when the straight equilibrium configuration begins to lose its stability, a stable steady-state response emerges. The stable steady-state response amplitude increases with the detuning parameter and the mean and the variation amplitude of the axial speed, decreases with the principal parametric resonance order, the dynamic viscosity, and the non-linear coefficients.

(4) Analytical study demonstrates that, when the unstable straight equilibrium configuration becomes stable again, an unstable steady-state response emerges. The unstable steady-state response amplitude increases with the detuning parameter, the dynamic viscosity, and the boundary constraint stiffness, decreases with the principal parametric resonance order, the variation amplitude of the axial speed, and the non-linear coefficient.

(5) Both analytical study and numerical calculations demonstrates that two non-linear models yield the qualitatively same results. Quantitatively, both models predict the same instability interval, while the non-linear integro-partial-differential equation leads to the larger amplitude of steady-state responses.

\section{Acknowledgments}

This work was supported by the National Outstanding Young Scientists Foundation of China (Project No. 10725209), the National Natural Science Foundation of China (Project Nos. 90816001 and 10902064), Shanghai Subject Chief Scientist Project (No. 09XD1401700), Shanghai Leading Talent Program, Shanghai Leading Academic Discipline Project (No. S30106), Shanghai Rising-Star Program (No. 11QA1402300), Innovation Program of Shanghai Municipal Education Commission (No. 12YZ028), and the program for Changjiang Scholars and Innovative Research Team in University (No. IRT0844).

\section{References}

[1] J.A. Wickert, Non-linear vibration of a traveling tensioned beam, Journal of Non-Linear Mechanics 27 (1992), $503-517$.

[2] F. Pellicano and F. Zirilli, Boundary layers and non-linear vibrations in an axially moving beam, International Journal of Non-Linear Mechanics 33 (1997), 691-711.

[3] B. Ravindra and W.D. Zhu, Low dimensional chaotic response of axially accelerating continuum in the supercritical regime, Archive of Applied Mechanics 68 (1998), 195-205.

[4] G. Chakraborty and A.K. Mallik. Parametrically excited nonlinear traveling beams with and without external forcing, Nonlinear Dynamics 17 (1998), 301-324.

[5] G. Chakraborty, A.K. Mallik and H. Hatwal, Non-linear vibration of a travelling beam, Journal of Non-Linear Mechanics 34 (1999), $655-670$.

[6] F. Pellicano and F. Vestroni, Nonlinear dynamics and bifurcations of an axially moving beam, ASME Journal of Vibration and Acoustics 122 (2000), 21-30.

[7] H.R. Öz, M. Pakdemirli and H. Boyaci, Non-linear vibrations and stability of an axially moving beam with time-dependent velocity, Journal of Non-Linear Mechanics 36 (2001), 107-115.

[8] R.G. Parker and Y. Lin, Parametric instability of axially moving media subjected to multifrequency tension and speed fluctuations, $A S M E$ Journal of Applied Mechanics 68 (2001), 49-57. 
[9] F. Pellicano, A. Fregolent, A. Bertuzzi and F. Vestroni, Primary and parametric non-linear resonances of a power transmission belt, Journal of Sound and Vibration 244 (2001), 669-684.

[10] K. Marynowski, Non-linear dynamic analysis of an axially moving viscoelastic beam, Journal of Theory Applied Mechanics 40 (2002), $465-482$.

[11] K. Marynowski and T. Kapitaniak, Kelvin-Voigt versus Bügers internal damping in modeling of axially moving viscoelastic web, Journal of Non-Linear Mechanics 37 (2002), 1147-1161.

[12] F. Pellicano and F. Vestroni, Complex dynamic of high-speed axially moving systems, Journal of Sound and Vibration 258 (2002), $31-44$.

[13] K. Marynowski, Non-linear vibrations of an axially moving viscoelastic web with time-dependent tension, Chaos, Solitons \& Fractals 21 (2004), 481-490.

[14] L.Q. Chen and X.D. Yang, Steady-state response of axially moving viscoelastic beams with pulsating speed: comparison of two nonlinear models, International Journal of Solids and Structure 42 (2005), 37-50.

[15] L.Q. Chen and W.J. Zhao, A conserved quantity and the stability of axially moving nonlinear beams, Journal of Sound and Vibration 286 (2005), 663-668.

[16] K.Y. Sze, S.H. Chen and J.L. Huang, The incremental harmonic balance method for nonlinear vibration of axially moving beams, Journal of Sound and Vibration 281 (2005), 611-626.

[17] S.H. Chen, J.L. Huang and K.Y. Sze, Multidimensional Lindstedt-Poincaré method for nonlinear vibration of axially moving beams, Journal of Sound and Vibration 306 (2007), 1-11.

[18] L.Q. Chen and X.D. Yang, Nonlinear free vibration of an axially moving beam: comparison of two models, Journal of Sound and Vibration 299 (2007), 348-354.

[19] K. Marynowski and T. Kapitaniak, Zener internal damping in modelling of axially moving viscoelastic beam with time-dependent tension, Journal of Non-Linear Mechanics 42 (2007), 118-131.

[20] W. Zhang and C.Z. Song, Higher-dimensional periodic and chaotic oscillations for viscoelastic moving belt with multiple internal resonances, International Journal of Bifurcation and Chaos 17 (2007), 1637-1660.

[21] M.H. Ghayesh and S.E. Khadem, Rotary inertia and temperature effects on non-nonlinear vibration, steady-state response and stability of an axially moving beam with time-dependent velocity, International Journal of Mechanics Sciences 50 (2008), 389-404.

[22] L.Q. Chen and H. Ding, Steady-state responses of axially accelerating viscoelastic beams: approximate analysis and numerical confirmation, Science in China Series G: Physics, mechanics and Astronomy 51 (2008), 1701-1721.

[23] M.H. Ghayesh and S. Balar, Non-linear parametric vibration and stability of axially moving visco-elastic Rayleigh beams, International Journal of Solids and Structure 45 (2008), 6451-6467.

[24] L.Q. Chen and B. Wang, Stability of axially accelerating viscoelastic beams: asymptotic perturbation analysis and differential quadrature validation, European Journal of Mechanics A/Solids 28 (2009), 786-791.

[25] H. Ding and L.Q. Chen, On Two Transverse Nonlinear Models of Axially Moving Beams, Science in China Series E: Technological Sciences 52 (2009), 743-751.

[26] L.Q. Chen and H. Ding, Steady-State Transverse Response in Coupled Planar Vibration of Axially Moving Viscoelastic Beams, ASME Journal of Vibration and Acoustics 132 (2010), 011009-1-011009-9.

[27] C.W. Bert and M. Malik, The differential quadrature method in computational mechanics: A review, Applied Mechanics Reviews 49 (1996), 1-28.

[28] C. Shu, Differential Quadrature and Its Application in Engineering. Springer, Berlin, 2001.

[29] L. Zhang and J.W. Zu, Nonlinear vibration of parametrically excited moving belts, ASME Journal of Applied Mechanics 66 (1999), $396-402$.

[30] E.M. Mockensturm and J. Guo, Nonlinear vibration of parametrically excited, viscoelastic, axially moving strings, ASME Journal of Applied Mechanics 72 (2005), 374-380.

[31] L.Q. Chen and X.D. Yang, Vibration and stability of an axially moving viscoelastic beam with hybrid supports, European Journal of Mechanics A/Solids 25 (2006), 996-1008.

[32] J.A. Wickert and C.D.Jr. Mote, Classical vibration analysis of axially moving continua, ASME Journal of Applied Mechanics 57 (1990), 738-744.

[33] L.Q. Chen and J.W. Zu, Solvability condition in multi-scale analysis of gyroscopic continua, Journal of Sound and Vibration 309 (2008), $338-342$.

[34] X. Wang and C.W. Bert, A new approach in applying differential quadrature to static and free vibration analyses of beams and plates, Journal of Sound and Vibration 162 (1993), 566-572. 

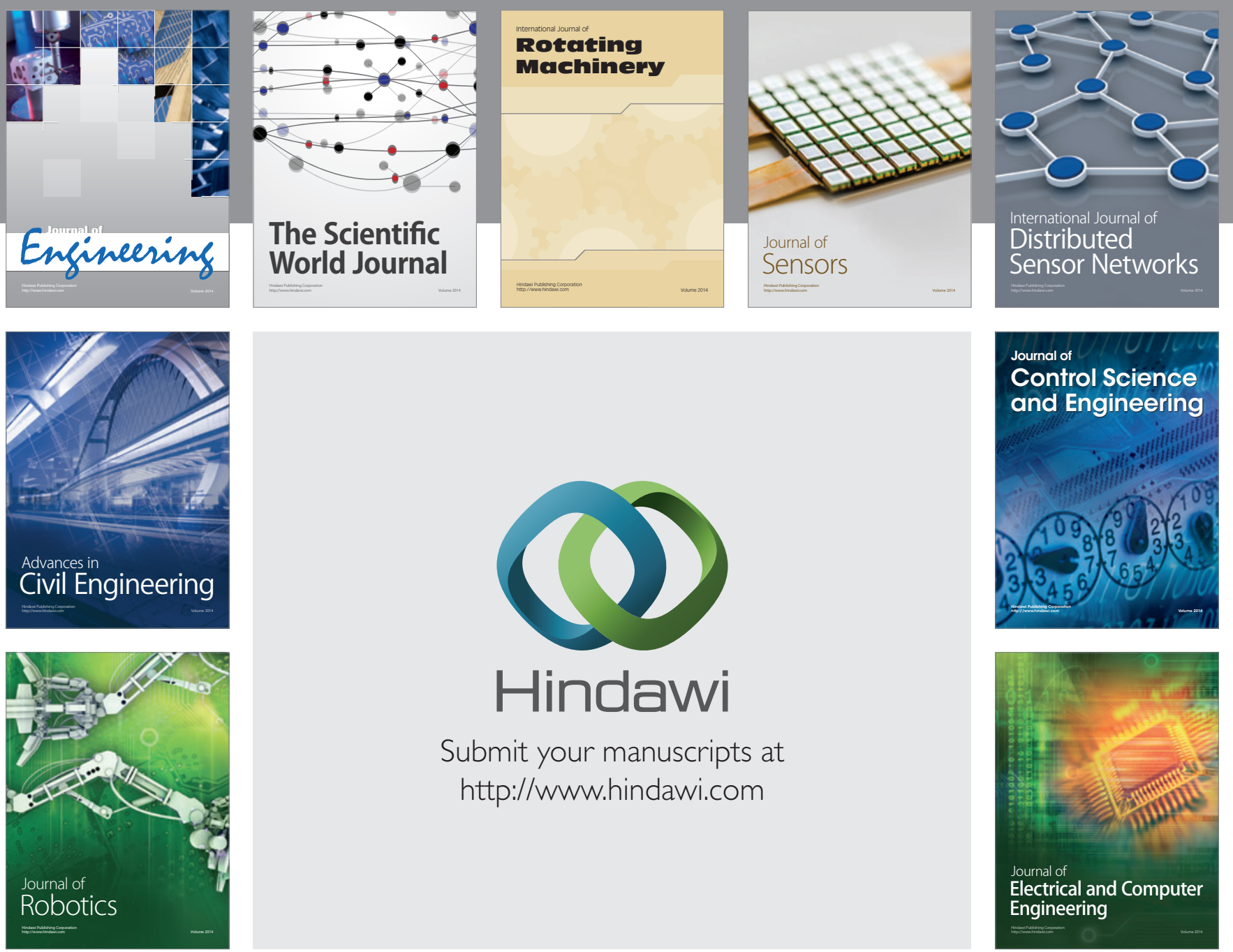

Submit your manuscripts at

http://www.hindawi.com
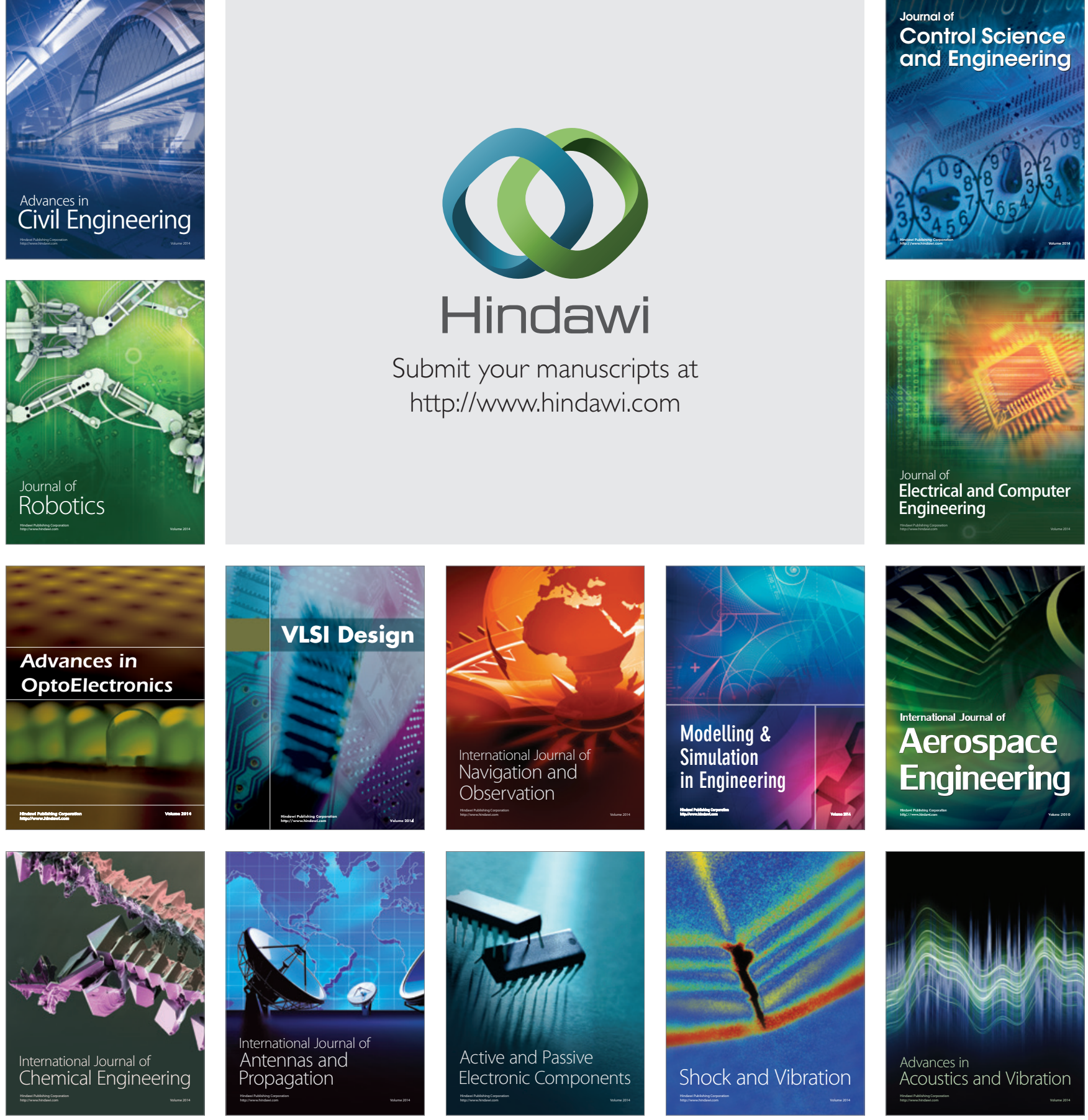